\title{
A COEFFICIENT PROBLEM WITH TYPICALLY REAL EXTREMAL FUNCTION
}

\author{
SEIJI KONAKAZAWA \\ (Communicated by Albert Baernstein II) \\ Dedicated to Professor Mitsuru Ozawa on the occasion of his 70 th birthday

\begin{abstract}
Let $\Sigma_{0}$ denote the class of univalent functions in $|z|>1$, with expansion $f(z)=z+\sum_{n=1}^{\infty} b_{n} z^{-n}$. We show that if the omitted set of an $f(z) \in \Sigma_{0}$ is on the trajectory arcs of the quadratic differential $-w(w-\lambda) d w^{2}$ with $\lambda \geqq 4(\sqrt{2}-1)$, then $f(z)$ has real coefficients. From this we can derive the coefficient estimate of $\max _{\Sigma_{0}} \mathscr{R} e\left(-b_{3}-\frac{1}{2} b_{1}^{2}+\lambda b_{2}\right)$.
\end{abstract}

\section{INTRODUCTION}

Let $\Sigma_{0}$ denote the class of functions, analytic and univalent in $|z|>1$, with expansion

$$
f(z)=z+\sum_{n=1}^{\infty} b_{n} z^{-n}
$$

We assume that the omitted set of an $f(z) \in \Sigma_{0}, \mathbb{C}-f(|z|>1)$, is on the trajectory arcs of the quadratic differential $Q(w) d w^{2}=\left(A_{0} w^{2}+A_{1} w+A_{2}\right) d w^{2}$ with $A_{0}, A_{1}, A_{2} \in \mathbb{R}$. We considered the sufficient conditions which imply that $f(z)$ has real coefficients [2]. In this paper, we study the case of $A_{2}=0$.

Now we suppose that the omitted set of $f(z) \in \Sigma_{0}$ is on the trajectory arcs of the quadratic differential $Q(w) d w^{2}=-w(w-\lambda) d w^{2}$ with real nonnegative $\lambda$. And the question is

\section{"Does $f(z)$ necessarily have real coefficients?"}

When $\lambda=0$, the answer is "No", because the quadratic differential is $-w^{2} d w^{2}$ in this case, and we readily know that the omitted set of $f(z) \in \Sigma_{0}$ is on the trajectory arcs of it when and only when

with $-1 \leqq c \leqq 1$

$$
f(z)=z\left(1+2 c i z^{-2}-z^{-4}\right)^{\frac{1}{2}}
$$

Received by the editors August 27, 1993 and, in revised form, January 17, 1994.

1991 Mathematics Subject Classification. Primary 30C50; Secondary 30C70.

Key words and phrases. Univalent function, quadratic differential. 
On the contrary, the answer is "Yes" provided $\lambda \geqq 4$, that is to say, $f(z)$ has real coefficients when $\lambda \geqq 4$. This is the case 3 ) of Theorem 1.5 in [2].

In the following section, we prove

Theorem. If $f(z) \in \Sigma_{0}$ has its omitted set on the trajectory arcs of the quadratic differential $-w(w-\lambda) d w^{2}$ with $\lambda \geqq 4(\sqrt{2}-1)$, then $f(z)$ has real coefficients.

Then we give the following related coefficient estimate.

\section{Corollary.}

$$
\begin{aligned}
& \operatorname{Max}_{\Sigma_{0}} \mathscr{R} e\left(-b_{3}-\frac{1}{2} b_{1}^{2}+\lambda b_{2}\right) \\
& = \begin{cases}\frac{\lambda^{4}}{64} \log \frac{4}{\lambda}-\frac{5}{256} \lambda^{4}+\frac{1}{4} \lambda^{2}+\frac{1}{2} & \text { if } 0<\lambda \leqq 4(\sqrt{2}-1), \\
\frac{\lambda^{4}}{64} \log \frac{\lambda+4+2 \sqrt{\lambda^{2}+2 \lambda+4}}{3 \lambda} & \\
+\left(\frac{17}{864} \lambda^{3}+\frac{7}{72} \lambda^{2}+\frac{1}{9} \lambda+\frac{4}{27}\right) \sqrt{\lambda^{2}+2 \lambda+4} & \text { if } 4(\sqrt{2}-1)<\lambda . \\
-\frac{17}{864} \lambda^{4}-\frac{4}{27} \lambda^{3}-\frac{2}{9} \lambda^{2}+\frac{8}{27} \lambda-\frac{11}{54} & \end{cases}
\end{aligned}
$$

This corollary gives the minimum value of $\mathscr{R} e\left(b_{3}+\frac{1}{2} b_{1}^{2}+\lambda b_{2}\right)$ by means of the rotation $-f(-z)$. Hence, with Theorem 2.2 of [2], we know the upper and lower sharp bounds of $\mathscr{R} e\left(b_{3}+\frac{1}{2} b_{1}^{2}+\lambda b_{2}\right)$.

\section{Proof of Theorem}

By making use of Schiffer's variational method [3], [4] we see that the associated quadratic differential with the coefficient problem

$$
\max _{\Sigma_{0}} \mathscr{R} e\left(-b_{3}-\frac{1}{2} b_{1}^{2}+\lambda b_{2}\right),
$$

which we study in the following section, is $-w(w-\lambda) d w^{2}$. Hence it is certain that there is an $f(z) \in \Sigma_{0}$ whose omitted set is on the trajectory arcs of the quadratic differential $-w(w-\lambda) d w^{2}$ for any $\lambda>0$. We fix such a function $f(z) \in \Sigma_{0}$. Then by the Schwarz reflection principle, we have

$$
\begin{aligned}
-f(z)(f(z)-\lambda) z^{2} f^{\prime}(z)^{2}= & -z^{4}+\lambda z^{3}+\left(2 b_{2}-\lambda b_{1}\right) z \\
& +\left(4 b_{3}-3 \lambda b_{2}+2 b_{1}^{2}\right)+\frac{2 b_{2}-\lambda b_{1}}{z}+\frac{\lambda}{z^{3}}-\frac{1}{z^{4}}
\end{aligned}
$$

where we know that the coefficient $2 b_{2}-\lambda b_{1}$ is real by the same consideration as in the proof of Case 1.3 of Theorem 1.5 in [2] which uses Jenkins' General Coefficient Theorem [1]. We note that the coefficient $4 b_{3}-3 \lambda b_{2}+2 b_{1}^{2}$ is also real since the right-hand side of $(1.1)$ is nonpositive on $|z|=1$. We denote the 
right-hand side of $(1.1)$ by $-z^{-4} q(z)$. Then, by $w=f(z),(1.1)$ becomes

$$
-w(w-\lambda) d w^{2}=-z^{-6} q(z) d z^{2} .
$$

Since $f(z)$ belongs to the class $\Sigma_{0}$, its conformal center $\frac{1}{2 \pi} \int_{0}^{2 \pi} f\left(e^{i \theta}\right) d \theta=$ 0 . Considering the global structure of the trajectories of $-w(w-\lambda) d w^{2}$, we know that the omitted set $\Gamma=f(|z|=1)$ contains the origin $w=0$ and forks in three directions there. We note that if $q\left(z_{0}\right)=0$ for some $z_{0},\left|z_{0}\right|>1$, then $q\left(\overline{z_{0}}\right)=0$ because $q(z)$ has real coefficients.

We suppose that the omitted set $\Gamma$ does not contain the critical point $w=\lambda$. Then we see by (1.2) that $q(z)$ has double zeros at $z=1, e^{i \alpha}$ and $e^{-i \alpha}$ for some real $\alpha$. and has simple zeros at $z=r$ and $\frac{1}{r}$ for some real $r>1$, and hence we have $q(r)=0$ and $f(r)=\lambda$. We put $J^{r}=\{x: x \geqq 1\}$. Then in this case, it follows from the relation (1.2) that $f(J)$ is a smooth Jordan arc which is always on trajectory or orthogonal trajectory arcs of the quadratic differential $-w(w-\lambda) d w^{2}$ and goes from $\Gamma$ to $\infty$ via $w=\lambda$. This is possible only when $f(J)$ is on the real axis. This means that $f(z)$ has real coefficients.

So let us see when the omitted set $\Gamma$ does not contain the critical point $w=\lambda$. In the opposite direction, we assume that $\Gamma$ contains $w=\lambda$. Then since $q(z)$ has real coefficients, there are four points $z=e^{i \alpha}, e^{-i \alpha}, e^{i \beta}$, and $e^{-i \beta}$, with some real $\alpha$ and $\beta$, which correspond to the tips of $\Gamma$ by $w=f(z)$ (when $w=\lambda$ is one of the tip points, we consider that $e^{i \beta}=e^{-i \beta}=1$ ). Hence we can rewrite $(1.1)$ as

$$
\begin{aligned}
-f(z) & (f(z)-\lambda) z^{2} f^{\prime}(z)^{2} \\
= & -z^{4}+\lambda z^{3}+\left(2 b_{2}-\lambda b_{1}\right) z+\left(4 b_{3}-3 \lambda b_{2}+2 b_{1}^{2}\right) \\
& +\frac{2 b_{2}-\lambda b_{1}}{z}+\frac{\lambda}{z^{3}}-\frac{1}{z^{4}} \\
= & -\frac{1}{z^{4}}\left[\left(z-e^{i \alpha}\right)\left(z-e^{-i \alpha}\right)\left(z-e^{i \beta}\right)\left(z-e^{-i \beta}\right)\right]^{2} .
\end{aligned}
$$

Thus we have

$$
\begin{aligned}
& z^{8}-\lambda z^{7}-\left(2 b_{2}-\lambda b_{1}\right) z^{5}-\left(4 b_{3}-3 \lambda b_{2}+2 b_{1}^{2}\right) z^{4}-\left(2 b_{2}-\lambda b_{1}\right) z^{3}-\lambda z+1 \\
& =\left[z^{4}-2(\cos \alpha+\cos \beta) z^{3}+2(1+2 \cos \alpha \cos \beta) z^{2}-2(\cos \alpha+\cos \beta) z+1\right]^{2} \\
& =z^{8}-4(\cos \alpha+\cos \beta) z^{7}+4\left((1+2 \cos \alpha \cos \beta)+(\cos \alpha+\cos \beta)^{2}\right) z^{6}+\cdots .
\end{aligned}
$$

By comparing coefficients we have

$$
\cos \alpha+\cos \beta=\frac{\lambda}{4} \quad \text { and } \quad \cos \alpha \cos \beta=-\frac{\lambda^{2}+16}{32}
$$

This gives us that $\cos \alpha, \cos \beta=\frac{\lambda \pm \sqrt{3 \lambda^{2}+32}}{8}$ and so it is necessary that $\frac{\lambda+\sqrt{3 \lambda^{2}+32}}{8} \leqq 1$. Hence we have that $\lambda \leqq 4(\sqrt{2}-1)$. It says that if the omitted set $\Gamma$ contains $w=\lambda$, then $\lambda \leqq 4(\sqrt{2}-1)$. Hence if $\lambda>4(\sqrt{2}-1)$, then $\Gamma$ cannot contain $\lambda$, so $f(z)$ must have real coefficients.

Now we suppose that $\lambda=4(\sqrt{2}-1)$. If the omitted set $\Gamma$ does not contain the critical point $\lambda$, then, as mentioned above, $f(z)$ has real coefficients. If 
$\Gamma$ contains $\lambda$, then in the above discussion $\cos \beta=\frac{\lambda+\sqrt{3 \lambda^{2}+32}}{8}=1$. This means that $z=1$ corresponds to $w=\lambda$ by $w=f(z)$. Hence the image of $J=\{x: x \geqq 1\}$ by $w=f(z)$ must be $[\lambda, \infty)$ on the positive real axis. Thus we know that $f(z)$ has real coefficients. This proves our Theorem.

\section{Proof of Corollary}

As we mentioned above, every extremal function $f(z)$ for the problem $\max _{\Sigma_{0}} \mathscr{R} e\left(-b_{3}-\frac{1}{2} b_{1}^{2}+\lambda b_{2}\right)$ must have its omitted set $\Gamma$ on the trajectory arcs of the quadratic differential $-w(w-\lambda) d w^{2}$. We divide our proof into two cases.

(I) First, we assume that $4(\sqrt{2}-1)<\lambda$. Then by Theorem, the extremal function $f(z)=z+\sum_{n=1}^{\infty} b_{n} z^{-n} \in \Sigma_{0}$ must have real coefficients. Moreover its proof tells us that its omitted set $\Gamma=f(|z|=1)$ does not contain the critical point $w=\lambda$. Hence we know that $\Gamma$ consists of three arcs emanating from the origin. Thus there are three points $z=1, e^{i \alpha}$ and $e^{-i \alpha}$, for some real $\alpha$, which correspond to the three tips of $\Gamma$ and the point $z=r$, for some real $r>1$, which corresponds to $w=\lambda$. By this setting, we have by the Schwarz reflection principle

$$
\begin{aligned}
- & f(z)(f(z)-\lambda) z^{2} f^{\prime}(z)^{2} \\
= & -z^{4}+\lambda z^{3}+\left(2 b_{2}-\lambda b_{1}\right) z+\left(4 b_{3}-3 \lambda b_{2}+2 b_{1}^{2}\right) \\
& +\frac{2 b_{2}-\lambda b_{1}}{z}+\frac{\lambda}{z^{3}}-\frac{1}{z^{4}} \\
= & -\frac{1}{z^{4}}\left[(z-1)\left(z-e^{i \alpha}\right)\left(z-e^{-i \alpha}\right)\right]^{2}(z-r)\left(z-\frac{1}{r}\right) .
\end{aligned}
$$

A comparison of coefficients gives that

$$
\begin{aligned}
& \lambda=4 \cos \alpha+2(1+R) \\
& \cos ^{2} \alpha+2(1+R) \cos \alpha+(1+R)=0, \\
& 2 b_{2}-\lambda b_{1}=4 \cos \alpha+2(1+R)\left(4 \cos ^{2} \alpha+3\right)+8(1+2 R) \cos \alpha
\end{aligned}
$$

and

$$
\begin{aligned}
-\left(4 b_{3}-3 \lambda b_{2}+2 b_{1}^{2}\right)= & 2+16(1+R) \cos \alpha \\
& +4(1+2 R)\left(2 \cos ^{2} \alpha+1\right),
\end{aligned}
$$

where $R=\frac{1}{2}\left(r+\frac{1}{r}\right)$.

It follows from (2.3) that $\cos ^{2} \alpha=-(1+R)(2 \cos \alpha+1)$. Hence we have

$$
\cos \alpha<-\frac{1}{2}
$$

By (2.2) and (2.3) we see that $\cos \alpha=\frac{\lambda-2 \pm \sqrt{\lambda^{2}+2 \lambda+4}}{6}$. Inequality (2.6) tells us that the negative sign is correct: 


$$
\cos \alpha=\frac{\lambda-2-\sqrt{\lambda^{2}+2 \lambda+4}}{6} .
$$

By (2.2) and (2.7) we also have

$$
R=\frac{\lambda-2+2 \sqrt{\lambda^{2}+2 \lambda+4}}{6}
$$

Now we integrate $(2.1)$ by using correspondence $\lambda=f(r)$,

$$
\begin{aligned}
& \int_{\lambda}^{w} \sqrt{w(w-\lambda)} d w \\
& \quad=\int_{r}^{z} \frac{1}{z^{3}}(z-1)\left(z^{2}-2 \cos \alpha \cdot z+1\right) \sqrt{(z-r)\left(z-\frac{1}{r}\right)} d z .
\end{aligned}
$$

Then it follows that

$$
\begin{gathered}
\frac{1}{4}(2 w-\lambda) \sqrt{w(w-\lambda)}-\frac{\lambda^{2}}{8} \log \{2 w-\lambda+2 \sqrt{w(w-\lambda)}\}+\frac{\lambda^{2}}{8} \log \lambda \\
=\frac{1}{4}\left\{F\left(z+\sqrt{(z-r)\left(z-\frac{1}{r}\right)}\right)-F(r)\right\}
\end{gathered}
$$

where

$$
\begin{aligned}
F(x)=\frac{x^{2}}{2}+a x-\frac{A}{(x-1)^{2}}-\frac{B}{x-1} & +E \log (x-1) \\
-\frac{p}{(x+1)^{2}}-\frac{q}{x+1}+t \log (x+1) & \quad-\frac{k}{2(x-R)^{2}}-\frac{m}{x-R}+n \log (x-R)
\end{aligned}
$$

with

$$
\begin{aligned}
& a=-4 \cos \alpha-R-2, \quad A=-2(R-1)^{2}, \quad p=2(R+1)^{2}, \quad k=\left(R^{2}-1\right)^{2}, \\
& B=-2(R-1)(4 \cos \alpha+R+1), \quad q=-2(R+1)(4 \cos \alpha+R+3), \\
& m=-2\left(R^{2}-1\right)(2 \cos \alpha+1) \text { and }-E=t=n=2(R+1)(4 \cos \alpha-R+3) .
\end{aligned}
$$

We substitute $w=z+\sum_{n=1}^{\infty} b_{n} z^{-n}$ into the left-hand side of (2.9) and expand both sides of it around $z=\infty$. Then by (2.7) and (2.8), a comparison of constant terms gives that

$$
\begin{aligned}
b_{1}= & -\frac{1}{12}\left(\lambda^{2}+8 \lambda+4\right)+\frac{1}{12}(\lambda+4) \sqrt{\lambda^{2}+2 \lambda+4} \\
& +\frac{\lambda^{2}}{8} \log \frac{\lambda+4+2 \sqrt{\lambda^{2}+2 \lambda+4}}{3 \lambda} .
\end{aligned}
$$

Hence by (2.4), (2.5), (2.7), (2.8) and (2.10) we obtain that

$$
\begin{aligned}
-b_{3}-\frac{1}{2} b_{1}^{2}+\lambda b_{2}= & \frac{\lambda^{4}}{64} \log \frac{\lambda+4+2 \sqrt{\lambda^{2}+2 \lambda+4}}{3 \lambda} \\
& +\left(\frac{17}{864} \lambda^{3}+\frac{7}{72} \lambda^{2}+\frac{1}{9} \lambda+\frac{4}{27}\right) \sqrt{\lambda^{2}+2 \lambda+4} \\
& -\frac{17}{864} \lambda^{4}-\frac{4}{27} \lambda^{3}-\frac{2}{9} \lambda^{2}+\frac{8}{27} \lambda-\frac{11}{54} .
\end{aligned}
$$


(II) Next we suppose that $0<\lambda \leqq 4(\sqrt{2}-1)$. In this case the omitted set $\Gamma=f(|z|=1)$ of the extremal function $f(z) \in \Sigma_{0}$ has the critical point $w=\lambda$ on it. In fact if $\Gamma$ does not contain $w=\lambda$, then, as we have seen in the proof of Theorem, the extremal function has real coefficients. And the relation (2.1) holds again. Then we have by $(2.8)$ that

$$
R=\frac{\lambda-2+2 \sqrt{\lambda^{2}+2 \lambda+4}}{6}>1
$$

since $R=\frac{1}{2}\left(r+\frac{1}{r}\right)>1$. And it follows that $4(\sqrt{2}-1)<\lambda$. This contradicts our current hypothesis. Hence the setting (1.3) holds in this case. Then by using (1.4) we know that the extremal function $w=f(z)$ satisfies the differential equation

$$
w(w-\lambda) d w^{2}=\frac{1}{z^{6}}\left[z^{4}-\frac{\lambda}{2} z^{3}-\frac{\lambda^{2}}{8} z^{2}-\frac{\lambda}{2} z+1\right]^{2} d z^{2} .
$$

We take square root of both sides of this and integrate them by using correspondence $\lambda=f\left(e^{i \theta}\right)$, for some real $\theta$,

$$
\int_{\lambda}^{w} \sqrt{w(w-\lambda)} d w=\int_{e^{i \theta}}^{z}\left(z-\frac{\lambda}{2}-\frac{\lambda^{2}}{8} z^{-1}-\frac{\lambda}{2} z^{-2}+z^{-3}\right) d z
$$

Then we have

$$
\begin{aligned}
\frac{1}{4}(2 w & -\lambda) \sqrt{w(w-\lambda)}-\frac{\lambda^{2}}{8} \log \{2 w-\lambda+2 \sqrt{w(w-\lambda)}\}+\frac{\lambda^{2}}{8} \log \lambda \\
& =\frac{1}{2} z^{2}-\frac{\lambda}{2} z-\frac{\lambda^{2}}{8} \log z+\frac{\lambda}{2} z^{-1}-\frac{1}{2} z^{-2}-i\left(\sin 2 \theta-\lambda \sin \theta-\frac{\lambda^{2}}{8} \theta\right) .
\end{aligned}
$$

We substitute $w=z+\sum_{n=1}^{\infty} b_{n} z^{-n}$ into the left-hand side and expand it around $z=\infty$, then it becomes

$$
\frac{1}{2} z^{2}-\frac{\lambda}{2} z-\frac{\lambda^{2}}{8} \log z+\left(b_{1}+\frac{\lambda^{2}}{16}+\frac{\lambda^{2}}{8} \log \frac{\lambda}{4}\right)+O\left(\frac{1}{z}\right) .
$$

Comparing the constant terms we have

$$
b_{1}=\frac{\lambda^{2}}{8} \log \frac{4}{\lambda}-\frac{\lambda^{2}}{16}+i \mu
$$

where $\mu$ is real. Then it follows from the relation (1.3) and (1.4) that

$$
b_{2}=\frac{\lambda}{2} b_{1}+\frac{\lambda}{2}-\frac{\lambda^{3}}{16} \quad \text { and } \quad b_{3}=\frac{3}{8} \lambda^{2} b_{1}-\frac{1}{2} b_{1}^{2}+\frac{\lambda^{2}}{4}-\frac{13}{256} \lambda^{4}-\frac{1}{2} .
$$

Hence we have

$$
\mathscr{R} e b_{2}=\frac{\lambda^{3}}{16} \log \frac{4}{\lambda}-\frac{3}{32} \lambda^{3}+\frac{\lambda}{2}
$$

and

$$
\mathscr{R} e b_{3}=-\frac{\lambda^{4}}{128}\left(\log \frac{4}{\lambda}\right)^{2}+\frac{7}{128} \lambda^{4} \log \frac{4}{\lambda}-\frac{39}{512} \lambda^{4}+\frac{\lambda^{2}}{4}-\frac{1}{2}+\frac{\mu^{2}}{2}
$$


Then it follows from (2.12), (2.13) and (2.14) that

$$
\mathscr{R} e\left(-b_{3}-\frac{1}{2} b_{1}^{2}+\lambda b_{2}\right)=\frac{\lambda^{4}}{64} \log \frac{4}{\lambda}-\frac{5}{256} \lambda^{4}+\frac{1}{4} \lambda^{2}+\frac{1}{2} \text {. }
$$

This completes the proof of Corollary.

\section{REMARKS}

In the proof of Corollary (I), we expand the left-hand side of (2.1). Then by using (2.4), (2.5) and (2.10), we know that all of the coefficients of the extremal function $f(z)=z+\sum_{n=1}^{\infty} b_{n} z^{-n}$ are represented in terms of $\lambda$. Hence the extremal function is unique and has real coefficients when $4(\sqrt{2}-1)<\lambda$. When $\lambda=4(\sqrt{2}-1)$ in the proof of Corollary (II), it follows from Theorem that the extremal function $f(z)$ has real coefficients. Thus by $(2.12)$ we know that $b_{1}$ is represented in terms of $\lambda$, and so a comparison of coefficients in (1.3) shows that the extremal function is unique and has real coefficients in this case. Hence the extremal function is unique and has real coefficients in the case of $4(\sqrt{2}-1) \leqq \lambda$ in Corollary.

On the other hand it is unsettled whether the extremal function is unique in the case of $0<\lambda<4(\sqrt{2}-1)$, in other words, whether Theorem is true for $0<\lambda<4(\sqrt{2}-1)$. Here we show the existence of the extremal function with real coefficients by direct construction.

Put $0<\lambda<4(\sqrt{2}-1)$. The mapping

$$
\begin{aligned}
\zeta & =\zeta(w)=\int_{\lambda}^{w} \sqrt{w(w-\lambda)} d w \\
& =\frac{1}{4}(2 w-\lambda) \sqrt{w(w-\lambda)}-\frac{\lambda^{2}}{8} \log [2 w-\lambda+2 \sqrt{w(w-\lambda)}]+\frac{\lambda^{2}}{8} \log \lambda
\end{aligned}
$$

carries the upper half plane $\mathscr{I} m w>0$, onto the domain bounded by the half line $\mathscr{I}_{m} \zeta=-\frac{\pi}{8} \lambda^{2}, \mathscr{R} e \zeta \geqq 0$, the segment $\mathscr{R} e \zeta=0,-\frac{\pi}{8} \lambda^{2} \leqq \mathscr{I} m \zeta \leqq 0$ and the positive real axis, the domain contains the point $\zeta=i$. On the other hand we take the mapping

$$
\begin{aligned}
\zeta=\zeta(z) & =\int_{1}^{z} \frac{1}{z^{3}}\left(z-e^{i \alpha}\right)\left(z-e^{-i \alpha}\right)\left(z-e^{i \beta}\right)\left(z-e^{-i \beta}\right) d z \\
& =\frac{1}{2} z^{2}-\frac{\lambda}{2} z-\frac{\lambda^{2}}{8} \log z+\frac{\lambda}{2} z^{-1}-\frac{1}{2} z^{-2}
\end{aligned}
$$

with $0<\beta<\alpha<\pi$ such that $\cos \alpha=\frac{\lambda-\sqrt{3 \lambda^{2}+32}}{8}, \cos \beta=\frac{\lambda+\sqrt{3 \lambda^{2}+32}}{8}$. Then it carries the domain $\mathscr{I} m z>0,|z|>1$, onto the domain whose boundary is the same as $\zeta(w)$ 's together with finite slits on the imaginary axis from $\zeta=0$ and $\zeta=-\frac{\pi}{8} \lambda^{2} i$. Hence we can combine these two mappings. Then by reflection we obtain the mapping of $|z|>1$ whose image domain is admissible with respect to the quadratic differential $-w(w-\lambda) d w^{2}$, and of course its expansion around $z=\infty$ has real coefficients. It satisfies the differential equation

$$
w(w-\lambda) d w^{2}=\left(z-\frac{\lambda}{2}-\frac{\lambda^{2}}{8} z^{-1}-\frac{\lambda}{2} z^{-2}+z^{-3}\right)^{2} d z^{2}
$$


Inserting $w=z+c_{0}+c_{1} z^{-1}+\cdots$ into the left-hand side of this, we know that $c_{0}=0$. Hence the mapping function belongs to the class $\Sigma_{0}$.

\section{REFERENCES}

1. J. A. Jenkins, Univalent functions and conformal mapping, Springer-Verlag, Berlin, 1958.

2. S. Konakazawa, Remarks on geometric properties of certain coefficient estimates, Kodai Math. J. 10 (1987), 242-249.

3. M. Schiffer, A method of variation within the family of simple functions, Proc. London Math. Soc. 44 (1938), 432-449.

4. G. Schober, Univalent functions-selected topics, Lecture Notes in Math., vol. 478, SpringerVerlag, New York, 1975.

Department of Mathematics, Tokyo National College of Technology, 1220-2 KunUGIDA-MACHI, HACHIOJI TOKYO 193, JAPAN

E-mail address: kona@tokyo-ct.ac.jp 